\title{
Nonlinear Micro-current Acquisition Device Applied to Onboard Langmuir Probe Instrument
}

\author{
Jin Wang, ${ }^{1,2}$ Qing-He Zhang, ${ }^{1 *}$ Qing-Fu Du, ${ }^{1,2}$ and Zan-Yang Xing ${ }^{1}$ \\ ${ }^{1}$ Institute of Space Sciences, Shandong University, No. 180, Wenhua West Rd, Weihai 264209, P.R. China \\ ${ }^{2}$ School of Mechanical, Electrical \& Information Engineering, Shandong University, \\ No. 180, Wenhua West Rd, Weihai 264209, P.R. China
}

(Received September 11, 2021; accepted December 13, 2021)

Keywords: Langmuir probe, onboard space electronics, micro-current acquisition, plasma diagnosis

An onboard Langmuir probe instrument equipped with a nonlinear micro-current acquisition device has been developed and is described in this paper. Langmuir probes are commonly used sensors for ionospheric plasma diagnosis, but the plasma density varies significantly with the altitude, latitude, and day/night; therefore, the currents collected by Langmuir probes have an extensive dynamic range and tiny amplitude. We developed a novel Langmuir probe instrument with a nonlinear micro-current acquisition device that can acquire micro-current signals with high precision and has an extended dynamic range. The instrument has a low mass (about $950 \mathrm{~g}$ ), a power consumption of about $700 \mathrm{~mW}$, and meets the payload requirements of micro-nano satellites. The instrument is equipped with high-speed analog-to-digital converters (ADCs) and a third-order low-pass filter to respectively achieve submeter spatial resolution and signal-tonoise ratio (SNR) measurement of higher than $120 \mathrm{~dB}$. A data inversion algorithm based on polynomial fitting is used to accurately trace back the collected current signals to achieve highprecision diagnosis of plasma in the density range from $10^{8}$ to $10^{13} \mathrm{~m}^{-3}$. The instrument has multiprobe acquisition capability, can run in two modes, and is suitable for different detection platforms. Various experiments were carried out in the Space Plasma Simulation Chamber to evaluate the performance of the instrument. The results demonstrate that the developed Langmuir probe instrument can accurately diagnose plasma in a large density range and meet the requirements for ionospheric plasma diagnosis.

\section{Introduction}

With increasing altitude, the upper terrestrial atmosphere becomes partially ionized by solar extreme ultraviolet (EUV) radiation, forming the ionosphere. Due to differences in the intensity of solar radiation and the influence of many other environmental factors, the plasma in the ionosphere exhibits irregular shapes on the spatial scale and variations in physical properties on the time scale. ${ }^{(1)}$ The size of a plasma cloud in the ionosphere varies from a few meters to several kilometers. ${ }^{(2,3)}$ The scale variation and physical properties of a plasma cloud reflect the variation 
of space weather, and changes in space weather are of great significance to modern society. Additionally, fluctuations in the ionosphere affect satellite-to-ground radio communications. ${ }^{(4)}$ Therefore, it is of great significance to study plasma in the ionosphere.

Langmuir probes have been widely used as diagnostic instruments for both laboratory and space plasmas due to their versatility, simplicity of construction, and the ease with which data can be acquired. ${ }^{(5-8)}$ Mott-Smith and Langmuir presented the orbital-motion-limited (OML) theory, ${ }^{(9)}$ which deals with collisionless electron and ion trajectories surrounding a spherical or cylindrical probe with radius much smaller than the Debye length. ${ }^{(10)}$ The Langmuir probe instrument has also been successfully applied to many exploration platforms as the main payload for space plasma diagnosis, especially on micro-nano satellites. The multi-needle Langmuir probe (m-NLP) instrument, invented by the University of Oslo, was successfully launched in 2017 onboard NorSat-1. ${ }^{(11)}$ The instrument works in a low-earth orbit (LEO) and can sample the electron density at a rate of up to $1 \mathrm{kSPS}$. The QB50 project within the European Union's Seventh Framework Programme for Research (FP7) aimed to launch a constellation of 50 CubeSats into the lower thermosphere at approximately $380 \mathrm{~km}$ altitude. ${ }^{(12)}$ The improved m-NLP had been deployed on three CubeSats in the QB50 project. To adapt to the limited resources in terms of size, power consumption, and data-link budget in the QB50 satellites, instrument designs have been adapted. This improved m-NLP can acquire micro-currents with a sampling frequency of up to 255 SPS. ${ }^{(12)}$ The China Seismo-Electromagnetic Satellite (CSES) was launched to detect the electromagnetic environment in space for the study of seismic early warning. ${ }^{(13)}$ A Langmuir probe is one of the payloads of the CSES, the first time that the Langmuir probe technique has been used in a Chinese satellite. This Langmuir probe instrument can detect plasma in the density range from $5 \times 10^{8}$ to $1 \times 10^{13} \mathrm{~m}^{-3}$. Because of the large size of the CSES, it carries two spherical probes with diameters of 50 and $10 \mathrm{~mm}$ with a large surface area, which creates favorable conditions for current collection. The DEMETER Langmuir probe instrument also comprises two Langmuir probe sensors: a cylindrical sensor (diameter $0.6 \mathrm{~cm}$, length $5 \mathrm{~cm}$, volume $1.4 \mathrm{~cm}^{3}$ ) and a segmented spherical sensor (sphere diameter $4 \mathrm{~cm}$, volume $\left.33.5 \mathrm{~cm}^{3}\right){ }^{(14)}$ However, the satellite acts as the counter electrode of the probe, and the small conductor area of the satellite will cause difficulties in the proper operation of spherical Langmuir probes. ${ }^{(15-17)}$ Thus, the Langmuir probes carried on micro-nano satellites are mainly cylindrical, and the current is much smaller than that of a spherical probe.

With the deepening of ionospheric research, small-scale plasma irregularities have attracted the attention of researchers. Therefore, a Langmuir probe instrument requires high spatial resolution and the capability of acquiring extremely small currents. This paper describes the mechanical and electronic design and functional testing of a Langmuir probe instrument built for micro-nano satellites. This instrument is equipped with a nonlinear micro-current acquisition device that can perform high-precision plasma diagnosis. 


\section{Theoretical Background and Design Requirements}

\subsection{Langmuir probe diagnostic method}

A Langmuir probe works by placing an exposed conductor in a plasma, biasing it relative to a reference potential, and measuring the collected current. ${ }^{(18)}$ Plasma parameters are determined by analysis of the probe's current-voltage $(I-V)$ characteristic. Therefore, the current acquisition accuracy of the probe system directly affects the reliability of the calculated plasma parameters.

A representative $I-V$ characteristic curve is shown in Fig. 1, where three operational regions of electron saturation, electron retardation, and ion saturation are separated by the plasma potential $\left(V_{p}\right)$ and the floating potential $\left(V_{f}\right) \cdot{ }^{(19)}$ Note that the magnitude of the ion current is exaggerated for illustration purposes.

In the electron saturation region, the ion current can be neglected due to the positive charge of the probe-repelling ions. The current collected by the cylindrical probe can be calculated as follows:

$$
I_{p}=I_{e}-I_{i} \approx I_{e}=N_{e} A e \sqrt{\frac{K_{B} T_{e}}{2 \pi m_{e}}} \frac{2}{\sqrt{\pi}} \sqrt{1+\frac{e\left(V_{R}-V_{p}\right)}{K_{B} T_{e}}},
$$

where $I_{p}$ is the probe current, $I_{e}$ is the current generated by the probe-absorbing electrons, $I_{i}$ is the current generated by the probe-absorbing ions, $N_{e}$ is the electron density, $A$ is the probe surface area, $e$ is the electron charge, $m_{e}$ is the electron mass, $K_{B}$ is Boltzmann's constant, $T_{e}$ is the electron temperature, $V_{R}$ is the voltage applied by the probe, and $V_{p}$ is the plasma potential. The square difference of Eq. (1) can be used to obtain the electron density with high spatial resolution, ${ }^{(20)}$ as shown in Eq. (2):

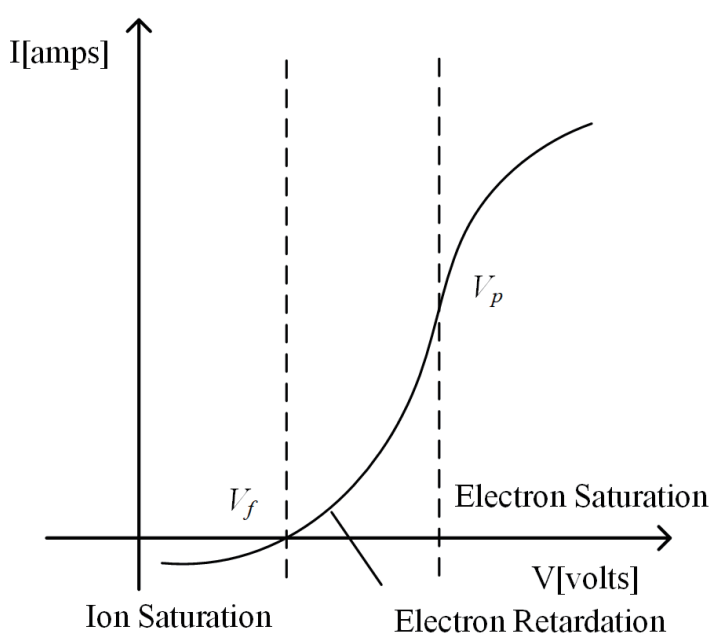

Fig. 1. Representative $I-V$ characteristic curve for a Langmuir probe. 


$$
N_{e}=\frac{1}{K A} \sqrt{\frac{\Delta\left(I_{p}{ }^{2}\right)}{\Delta V_{R}{ }^{2}}},
$$

where $K=\left(e^{3 / 2} / \pi\right) \sqrt{2 / m_{e}}$. Using Eq. (2), it is possible to quickly calculate the electron density using two points in the electron saturation region of the $I-V$ characteristic or two probes set in the saturation region with different fixed bias voltages. ${ }^{(21)}$ This creates suitable conditions for determining the boundary of a small-scale plasma cloud in space. However, to obtain parameters such as the electron temperature, it is necessary to obtain the complete curve. The plasma quantities of $T_{e}, V_{f}$, and saturation current $I_{\text {sat }}$ are determined by fitting this model function to the measured characteristic. ${ }^{(22)}$ The electron temperature $\left(T_{e}\right)$ can be obtained from the electron density $\left(N_{e}\right)$ and the current $\left(I_{s a t}\right)$ at the separation point between the electron retardation region and electron saturation region using the following equation:

$$
I_{s a t}=N_{e} A e \sqrt{\frac{K_{B} T_{e}}{2 \pi m_{e}}} .
$$

\subsection{Langmuir probe characterization}

Plasma exhibits electrical neutrality from a macroscopic viewpoint, but an electric field exists near each charged particle. When the electric field is shielded by the field of surrounding particles, it appears electrically neutral outside a certain space. This phenomenon is called Debye shielding, ${ }^{(23)}$ and the size of the space occupied by the particle shielding field is called the Debye length $\lambda_{B}$. In the shielding field generated by the particles, that is, in the 3D sphere around the particles, the condition of plasma neutrality is not satisfied.

The plasma boundary can be divided into three parts, the sheath, presheath, and plasma, with increasing distance from the vessel wall (plasma boundary). As shown in Fig. 2, the interior of

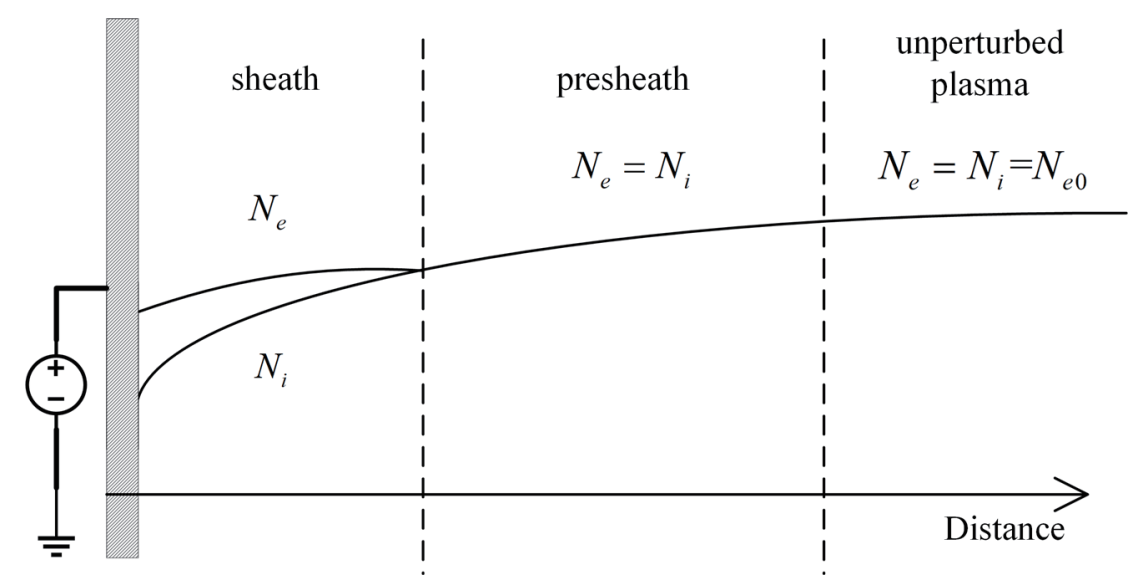

Fig. 2. Density and potential probes of non-neutral regions adjacent to a wall that is biased positively relative to the plasma potential. The sheath thickness is on order of the Debye scaling length. 
the sheath does not satisfy electrical neutrality, and the thickness of the sheath $r_{s}$ is on the order of the Debye length $\lambda_{B}{ }^{(23)}$

Theoretically, there is no general analytic expression for the collected current for all Langmuir probe geometries, plasma conditions, spacecraft velocities, and probe orientations. Therefore, probes are usually designed for special limiting cases such as OML collection, which is only applicable when the probe characteristic size is significantly smaller than the Debye length. The characteristic size of the probe is calculated as follows:

$$
d \approx r_{P} \ln \left(\frac{\pi l_{p}}{4 r_{p}}\right),
$$

where $r_{p}$ is the radius of the probe and $l_{p}$ is the probe length. We take the characteristic size of the probe to be less than 0.1 Debye length as the design condition and determine $r_{p}$ to be 0.25 $\mathrm{mm}$ and $l_{p}$ to be $25 \mathrm{~mm}$. Under these conditions, the characteristic size of the cylindrical probe is calculated to be approximately $1.1 \mathrm{~mm}$, which meets the theoretical requirements of OML theory. The design of the cylindrical Langmuir probe is shown in Fig. 3.

\subsection{Electronics design requirements}

When the satellite operates in a LEO above $300 \mathrm{~km}$, the electron density varies from $10^{9}$ to $10^{13} \mathrm{~m}^{-3}$ and the electron temperature ranges from 800 to $4000 \mathrm{~K}$. Combining these values, the probe current can be roughly calculated as nA to $\mu \mathrm{A}$.

When the Langmuir probe is installed on a micro-nano satellite, the satellite acts as the counter electrode of the Langmuir probe, and its conductive area must be at least $\sim 100$ times larger than the probe electrode surface area for correct $T_{e}$ measurement and $\sim 1000$ times larger to measure $N_{e}$ correctly. ${ }^{(15)}$ Therefore, the size of the satellite limits the surface area of the probe, resulting in a small amount of current collected.

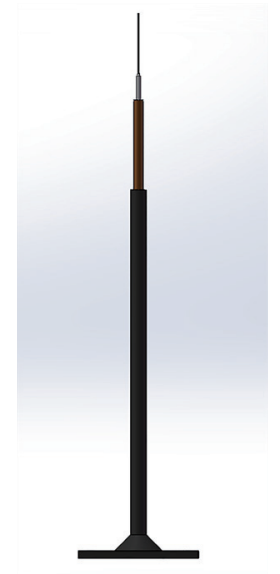

Fig. 3. (Color online) Typical Langmuir probe with $25 \mathrm{~mm}$ length and $0.51 \mathrm{~mm}$ diameter. 
It is difficult for the linear amplifier circuit to realize the precise acquisition and amplification of such an extensive dynamic range of micro-currents. Thus, we develop a Langmuir probe instrument to measure both $T_{e}$ and $N_{e}$ that can satisfy the requirements for micro-nano satellites: (1) the instrument can obtain $N_{e}$ with high spatial resolution reaching the submeter level; (2) the instrument can collect micro-currents with an extended dynamic range and has the ability to detect changes in space weather, such as magnetic storms; (3) the instrument should be compact, have low power consumption, and be able to work with micro-nano satellite conductive areas.

\section{Instrument Design}

To accurately process the weak current signal collected by the Langmuir probe, a nonlinear amplifier circuit based on the logarithmic operation is designed to process the signal at the front end of analog-to-digital converters (ADCs). In the weak-current area, logarithmic amplification is mainly used with high sensitivity; in the large-current area, linear amplification is used to take into account the current collection.

\subsection{Design of nonlinear micro-current amplifier circuit}

The $I-V$ relationship between the two ends of the PN junction [Eq. (5)] can be used to realize the logarithmic operation of the current signal.

$$
i_{P N}=I_{S}\left(e^{\left(q V_{P N} / K_{B} T\right)}-1\right)
$$

Here, $I_{S}$ is the reverse saturation current, $K_{B}$ is Boltzmann's constant, $V_{P N}$ is the voltage across the PN junction, $i_{P N}$ is the current flowing through the PN junction, and $V_{T}=K_{B} T / q$; when $T=$ $300 \mathrm{~K}, V_{T}=26 \mathrm{mV}$. The temperature in the satellite cabin generally fluctuates within $300 \pm 10 \mathrm{~K}$ under the adjustment of the thermal control system, and the impact on $V_{T}$ does not exceed \pm 1 $\mathrm{mV}$.

When $V_{P N} \gg V_{T}$, the above equation can be simplified as follows:

$$
V_{P N} \approx V_{T} \ln \left(\frac{i_{P N}}{I_{S}}\right)
$$

In the circuit design, we use the PN junction on the bipolar junction transistor (BJT) as the feedback path of the op-amp, so that a larger dynamic range of the conversion current can be obtained. After test experiments on the PN junctions of various types of BJT, the SS9012 transistor is selected for the circuit design owing to its excellent performance. This is a PNP transistor suitable for radio receivers, its collector current is $-500 \mathrm{~mA}$, and it has an excellent direct current amplification capability.

We have designed a nonlinear amplifier circuit based on the logarithm operation that eliminates the influence of temperature changes on the amplifier circuit and increases the robustness of the circuit. The compensated nonlinear amplifier circuit is shown in Fig. 4. ${ }^{(24)}$ 


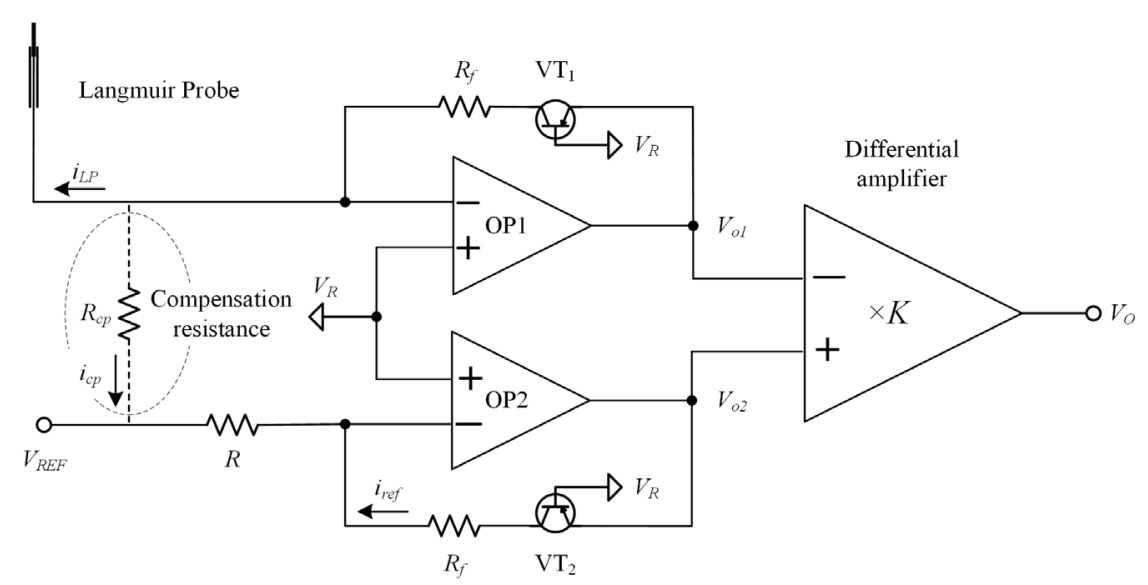

Fig. 4. Circuit diagram of nonlinear amplifier circuit based on logarithm operation.

The Langmuir probe is connected to the inverting input (omitting the long cables) of the opamp (OP1). The high-performance JFET input op-amp OPA4196 is chosen for the circuit design due to its low input bias current of $\pm 5 \mathrm{pA}$. Regarding the selection of resistors, there are few requirements, but resistors with negative temperature coefficients will compensate part of the BJT's error caused by temperature changes, although this error is relatively small.

$V_{R}$ is the scanning voltage applied to the probe, which is loaded on the probe through the principle that the non-inverting input terminal and the inverting input terminal of the op-amp have the same potential. Moreover, the base potentials of the two BJTs are also $V_{R}$.

Without considering the existence of compensation resistance, the probe current flows through $\mathrm{VT}_{1}$ and the resistance $R_{f}$ to induce a voltage drop. Therefore, the output voltage $\left(V_{o 1}\right)$ of OP1 relative to the scanning voltage $V_{R}$ is the result of the linear and nonlinear superpositions of current flowing through resistors $R_{f}$ and $\mathrm{VT}_{1}$, respectively. The output voltage of OP1 is as follows:

$$
V_{o 1}=i_{L P} \cdot R_{f}+V_{T} \ln \left(\frac{i_{L P}}{I_{S}}\right)+V_{R}
$$

Similarly, the output voltage $\left(V_{o 2}\right)$ of OP2 can be determined as

$$
V_{o 2}=i_{r e f} \cdot R_{f}+V_{T} \ln \left(\frac{i_{r e f}}{I_{S}}\right)+V_{R}
$$

where $i_{\text {ref }}$ is the current flowing through $\mathrm{VT}_{2}$ and $i_{\text {ref }}$ is obtained as

$$
i_{\text {ref }}=\frac{V_{R}-V_{R E F}}{R}
$$

By setting $V_{R E F}$, we can ensure that $i_{r e f}$ is a reasonable value. 
The differential amplifier subtracts the output voltages of OP1 and OP2 to eliminate $V_{R}$ and $I_{S}$, reduce the impact of temperature changes on the output, and make the following analog-todigital (A/D) conversion more convenient.

The relationship between the output voltage of the differential amplifier $\left(V_{o}\right)$ and the input current $\left(i_{L P}\right)$ is as follows:

$$
\begin{aligned}
V_{o} & =K\left(V_{o 2}-V_{o 1}\right) \\
& =K\left[\begin{array}{l}
\left.V_{R}+i_{r e f} \cdot R_{f}+V_{T} \ln \left(\frac{i_{r e f}}{I_{S}}\right)-V_{R}\right] \\
-i_{L P} \cdot R_{f}-V_{T} \ln \left(\frac{i_{L P}}{I_{S}}\right)
\end{array}\right], \\
& =K\left[V_{T} \ln \left(\frac{i_{r e f}}{i_{L P}}\right)+R_{f}\left(i_{r e f}-i_{L P}\right)\right]
\end{aligned}
$$

where $K$ is the gain of the differential amplifier and $V_{R}$ is the scanning voltage loaded on the probe. As stated above, through the calculation of the differential amplifier circuit, the influence of temperature changes on $I_{S}$ can be eliminated.

On the other hand, when the current through the PN junction is greater than $2 \mu \mathrm{A}$, the slope of the voltage change across the PN junction is small, indicating that the change in voltage with the increase in the collected current is too gradual, which may cause the problem of poor accuracy for the collection of higher currents. We experimentally verified that the dynamic performance at higher currents can be improved by connecting a resistor $\left(R_{f}\right)$ in series with the BJTs $\left(\mathrm{VT}_{1}\right.$ and $\left.\mathrm{VT}_{2}\right)$.

When the scanning voltage is lower than $V_{f}$, the current is reversed, and the circuit must measure the reverse current. To solve the problem that the PN junction of the BJT cannot be effectively measured when the current flow changes, a compensation resistance is added to realize the ion current measurement. A compensation resistor $R_{c p}$ is connected to $V_{R E F}$ at the inverting input of the OP1, then the acquisition zero point can be moved negatively by adjusting $V_{R E F}$. We set $R_{c p}=R$, i.e., $i_{c p}=i_{\text {ref }}$. After compensation, the relationship between the output voltage $V_{o c p}$ and the input current $i_{L P}$ is given by

$$
\begin{aligned}
V_{o c p} & =K\left[V_{T} \ln \left(\frac{i_{r e f}}{i_{L P}+i_{c p}}\right)+R_{f}\left(i_{r e f}-i_{L P}-i_{c p}\right)\right] \\
& =K\left[V_{T} \ln \left(\frac{i_{r e f}}{i_{L P}+i_{r e f}}\right)-i_{L P} R_{f}\right]
\end{aligned}
$$




\subsection{Design of low-pass filter circuit}

The Langmuir probe works in space plasma or a plasma chamber, but the acquisition device is generally under normal conditions in the satellite or outside the plasma chamber. Therefore, a long connecting wire is needed to connect the probes to the acquisition device. Due to the interference of radio waves in the environment, a long connection wire will superimpose highfrequency noise on the collected direct currents, resulting in more significant noise in the amplified signal. To improve the signal-to-noise ratio (SNR) of the collected signal, the amplified signal must be subjected to low-pass filtering.

An integrated low-pass filter (LPF) chip has excellent performance, and the cutoff frequency can be configured through simple peripheral circuits. However, it has the disadvantage of high power consumption. Considering the strict requirements of the satellite payload in terms of power consumption, a third-order LPF composed of a single op-amp is used. The third-order LPF circuit diagram is shown in Fig. 5.

A differential equation is established for each node of the LPF circuit, and the frequency domain model is obtained as follows:

$$
W(s)=\frac{V_{o L P F}(s)}{V_{i}(s)}=\frac{-k}{a_{0} S^{3}+a_{1} S^{2}+a_{2} S+a_{3}}
$$

where $k=\frac{R_{3}}{R_{1}+R_{2}}, a_{0}=\frac{R_{1} R_{2} R_{3} R_{4} C_{1} C_{2} C_{3}}{R_{1}+R_{2}}, a_{1}=\frac{R_{1} R_{2} R_{3} R_{4} C_{3}}{R_{1}+R_{2}}\left[C_{1}\left(\frac{1}{R_{2}}+\frac{1}{R_{3}}+\frac{1}{R_{4}}\right)+C_{2}\left(\frac{1}{R_{1}}+\frac{1}{R_{2}}\right)\right]$, $a_{2}=\frac{C_{1} R_{1} R_{2}+C_{3} R_{3} R_{4}}{R_{1}+R_{2}}+C_{3}\left(R_{3}+R_{4}\right), a_{3}=1$.

The cutoff frequency of the LPF is generally set to $100 \mathrm{~Hz}$ to prevent switching noise of the switching power supply integrated circuit, which is usually greater than $20 \mathrm{kHz}$. In this case, $R_{1}=R_{2}=R_{4}=15 \mathrm{k} \Omega, R_{3}=30 \mathrm{k} \Omega, C_{1}=C_{2}=100 \mathrm{nF}$, and $C_{3}=10 \mathrm{nF}$. However, in the experiment, we generally set the cutoff frequency below $50 \mathrm{~Hz}$ to prevent the $50 \mathrm{~Hz}$ interference of the power supply system.

The logarithmic amplitude-frequency characteristics and logarithmic phase-frequency characteristics of the configured filter circuit are respectively shown in Figs. 6(a) and 6(b). To

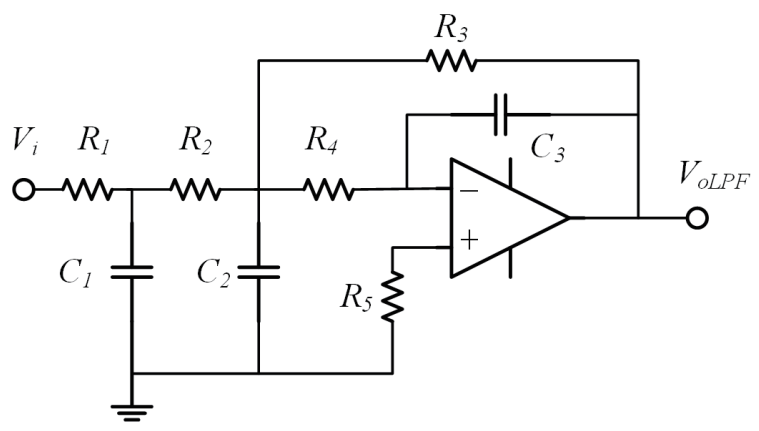

Fig. 5. Circuit diagram of third-order LPF. 
test the performance of the filter, Gaussian white noise, uniform white noise, and high-frequency sinusoidal noise are superimposed on the low-frequency sinusoidal signal with 9000 data points. A comparison of the signal before and after it passes through the LPF is shown in Fig. 6(c). The filter effectively limits high-frequency noise.

The relationship between the output signal after the filter and the input current is

$$
V_{o L P F}=K\left[V_{T} \ln \left(1+\frac{i_{L P}}{i_{r e f}}\right)+R_{f} i_{T Z}\right],
$$

where $i_{\text {ref }}$ is the maximum value of the ion current that can be collected. It can be seen from Eq. (13) that when $i_{L P} \rightarrow-i_{r e f}, V_{o L P F}$ tends to infinity. However, through the instrument's microcontroller unit (MCU), $V_{R E F}$ can be dynamically adjusted to enable the collection of plasma ion currents with different densities. When collecting ion current, that is, when $i_{L P}<0$ and $V_{O L P F}<0$, to adapt to the ADCs that can only convert positive voltage, the output voltage can be increased by $V_{b}$ before A/D conversion.

The relationship between the output voltage $\left(V_{O N C A}\right)$ and the probe current $\left(i_{L P}\right)$ of the nonlinear current acquisition (NCA) circuit is

$$
V_{o N C A}=K\left[V_{T} \ln \left(1+\frac{i_{L P}}{i_{r e f}}\right)+R_{f} i_{L P}\right]+V_{b}
$$

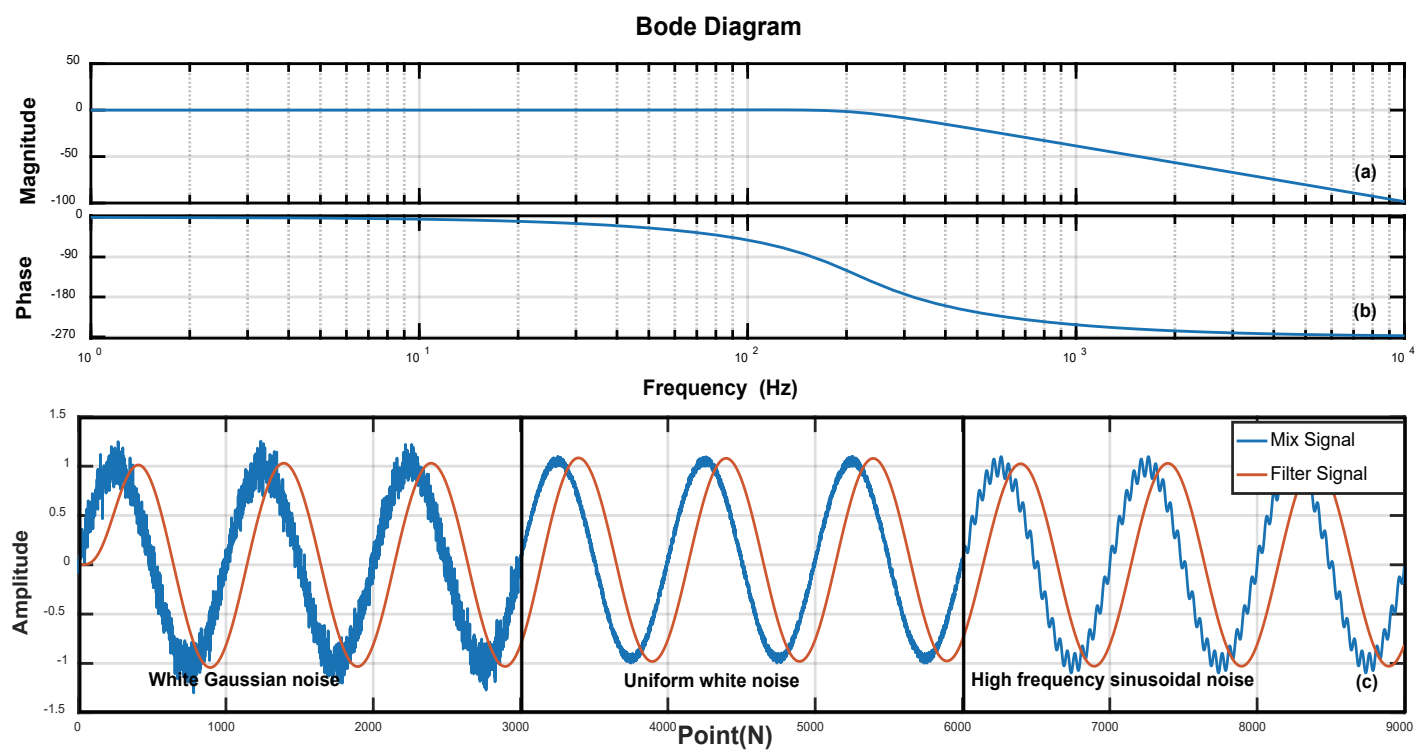

Fig. 6. (Color online) (a) Logarithmic amplitude-frequency curve of filter. (b) Logarithmic phase-frequency curve of filter. (c) Contrast between the input signal and output signal of the filter. 


\subsection{Overall system design}

The probe current signal is converted into a voltage signal by the NCA circuit and directly input to the ADCs for voltage acquisition. Different from the simulation environment of the plasma chamber, the satellite has a high speed when it orbits the earth. To achieve high-spatialresolution measurement to detect the fine structure of the plasma, the sampling frequency must be increased. ${ }^{(19)}$ Using an A/D conversion module with a built-in MCU can simplify the design to achieve a sampling frequency $\left(f_{\text {samp }}\right)$ of $10 \mathrm{kSPS}$. From $f_{\text {samp }}$ and the satellite speed $\left(V_{\text {sat }}\right)$, the spatial resolution $\left(R_{\text {spa }}\right)$ can be obtained from Eq. (15), which meets the requirement of submeter spatial resolution.

$$
R_{\text {Spa }}=\frac{V_{\text {Sat }}}{f_{\text {Samp }}}
$$

The functional block diagram of the instrument is shown in Fig. 7.

The instrument can collect currents from four Langmuir probes. The MCU can be used to set the probe in scan mode or fixed bias mode. The scan mode is used to obtain a complete curve to determine parameters such as electron temperature, and the two probes in the fixed bias mode are set to different voltages to achieve high-spatial-resolution sampling of ionospheric plasma.

Satellites have strict requirements on the mass and power consumption of the instruments, especially for micro-nano satellites. The mass and power consumption of various previous instruments are relatively large. The Ionospheric Plasma and Electrodynamics Instrument (IPEI) is one of the three payloads on the ROCSAT-1 satellite. ${ }^{(25)}$ The total mass of the IPEI, including the cable, is $9.26 \mathrm{~kg}$. The Advanced Ionospheric Probe (AIP) on the FORMOSAT-5 satellite has a mass of $4.5 \mathrm{~kg} .{ }^{(26)}$ The Compact Ionosphere Probe (CIP) on the Ionospheric Dynamics Exploration and Attitude Subsystem Satellite (IDEASSat/INSPIRESat-2) has a power consumption of about $3.5 \mathrm{~W} .{ }^{(27)}$ Compared with previous instruments, the Langmuir probe instrument described in this paper has a lower power consumption $(750 \mathrm{~mW})$ and mass (about $950 \mathrm{~g})$.

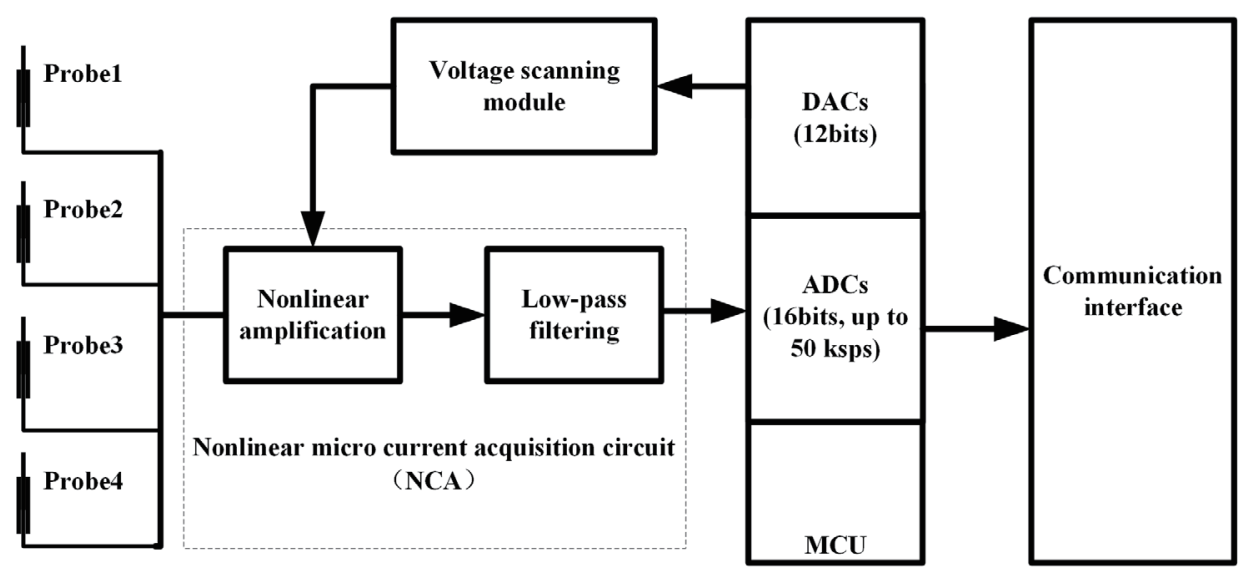

Fig. 7. Block diagram of instrument function. 


\section{Data Inversion Method}

As shown in Eq. (14), the NCA's output voltage is the superposition of the logarithmic and linear terms. It is difficult to trace the variables by solving the equation, which increases the difficulty of inverting the probe current. Because we already know the theoretical relationship between the output voltage and the probe current, we can use high-order polynomial fitting to obtain the probe current from the measured voltage.

The theoretical output curve and the amplification relationship of the NCA circuit are shown in Fig. 8. It can be seen that the amplifier circuit has a significant amplifying effect on weak signals and can markedly improve the SNR of weak signals. According to the corresponding magnification of the collected currents, the absolute magnitude of the collected current can be accurately inverted according to the voltage on the ADCs.

\section{Experimental Setup and Results}

\subsection{Experimental setup}

The plasma chamber installed in the Space Plasma Detection Laboratory (SPDL) can reach a background vacuum of $10^{-5} \mathrm{~Pa}$. The plasma was created by impact ionization using electrons emitted from a negatively biased and heated filament at the bottom of the chamber. ${ }^{(28)}$ The entire system can simulate low-density plasma in the ionosphere, and the minimum plasma density that can be stably maintained is about $1 \times 10^{11} \mathrm{~m}^{-3}$. A sketch of the experimental setup is shown in Fig. 9.

\subsection{Experimental analysis}

The experiment was carried out under a pressure of $0.09 \mathrm{~Pa}$, and the plasma density was adjusted by changing the discharge filament current while keeping the pressure constant.

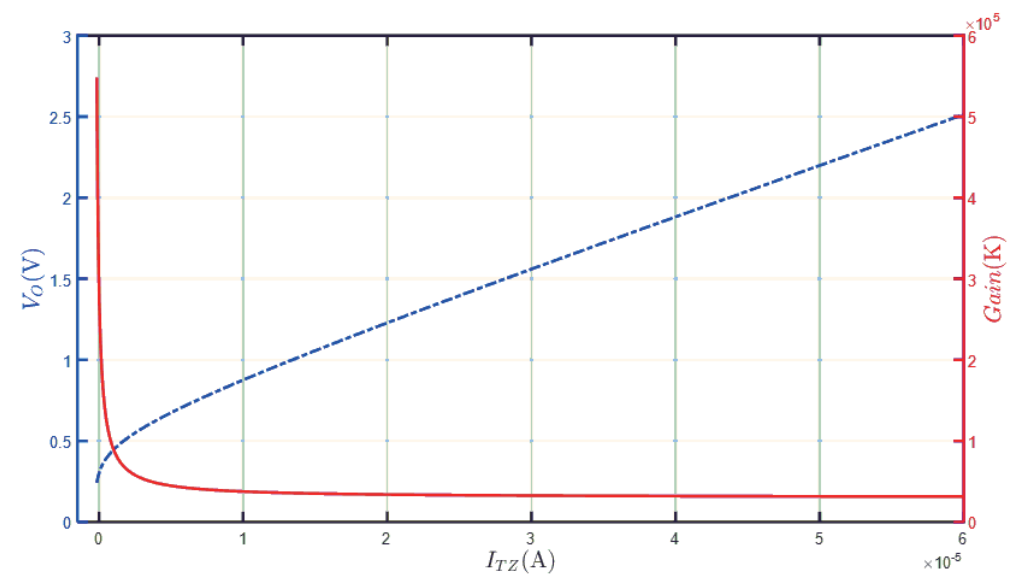

Fig. 8. (Color online) Theoretical output curve and amplification relationship of NCA circuit. 


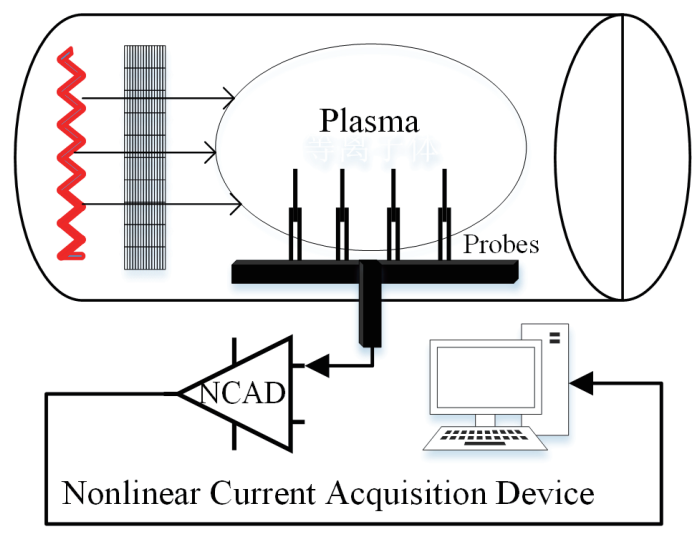

Fig. 9. (Color online) Sketch of experimental setup.
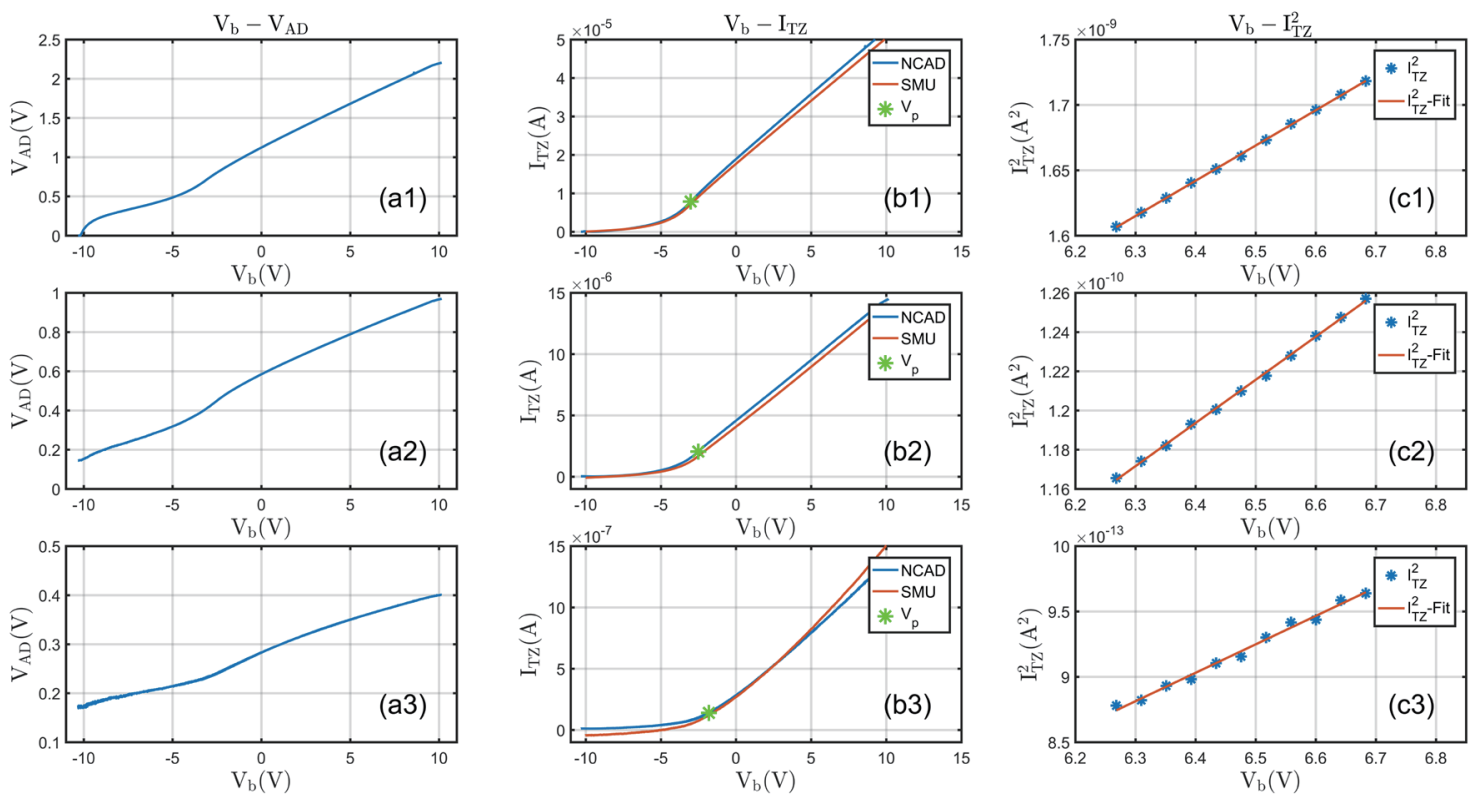

Fig. 10. (Color online) (a) Voltage acquisition at ADCs. (b) Comparison of collected data. (c) Square of probe current vs probe bias potential.

Multiple sets of experiments to collect $I-V$ characteristic curves were carried out within the plasma density range from $10^{11}$ to $10^{13} \mathrm{~m}^{-3}$, and the experimental results are shown in Fig. 10.

Figure 10(a) shows the collected port voltage of the ADCs and Fig. 10(b) shows a comparison of the current collected by a source measurement unit (SMU) and the current collected by a nonlinear current acquisition device (NCAD). The results of experiments performed at plasma densities of $10^{11}-10^{13} \mathrm{~m}^{-3}$ are shown. Due to the increase in the sheath thickness and the influence of the edge on the current collected by the probe, the curve does not clearly show saturation behavior. However, the boundary point between the electron retardation region and the electron saturation region can be defined by finding the maximum value of the derivative of the $I-V$ curve, as marked in Fig. 10(b). Figure 10(c) shows the linear relationship between the 
Table 1

Correlation and normalized residual square of sums of NCAD and MCU acquisition curves in different density ranges.

\begin{tabular}{lcc}
\hline Plasma density $\left(\mathrm{m}^{-3}\right)$ & Correlation & Normalized residual sum of squares \\
\hline $2.3 \times 10^{13}$ & 0.9998 & $1.5230 \times 10^{-4}$ \\
$1.6 \times 10^{13}$ & 0.9996 & $1.8817 \times 10^{-4}$ \\
$1.4 \times 10^{13}$ & 0.9999 & $7.5384 \times 10^{-5}$ \\
$9.8 \times 10^{12}$ & 0.9999 & $6.6589 \times 10^{-5}$ \\
$4.1 \times 10^{12}$ & 1.0000 & $2.7578 \times 10^{-5}$ \\
$1.2 \times 10^{12}$ & 0.9997 & $7.6457 \times 10^{-6}$ \\
$4.7 \times 10^{11}$ & 0.9992 & $4.6285 \times 10^{-6}$ \\
$2.6 \times 10^{11}$ & 0.9995 & $7.3329 \times 10^{-6}$ \\
$1.1 \times 10^{11}$ & 0.9977 & $2.8672 \times 10^{-5}$ \\
\hline
\end{tabular}

square of the collected current and the voltage in the electron saturation region. The electron density can be quickly calculated from the slope of the curve. ${ }^{(10)}$

By comparing the data obtained from the experiment, it can be concluded that when the plasma has a medium or high density, the current collected by the NCAD is slightly larger than the current collected by the SMU. The opposite result is obtained when the plasma density is lower, but the overall error is small. The error is small at high plasma densities.

Next, nine sets of comparison curves were obtained under different density conditions, and their correlation and the normalized residual square sum were calculated, as shown in Table 1. The residual sum of squares of the evaluation index of the fitted curve is also introduced to evaluate the consistency of the two curves. The residual sum of squares is given by $e_{S S E}=\sum_{i=1}^{n}\left(x_{i}-y_{i}\right)^{2}$, where $x_{i}$ and $y_{i}$ are the corresponding data points of the two sets of comparative data. A smaller residual sum of squares indicates that the two curves are more consistent. However, due to the different dimensions of each group of data, simply comparing the residual sum of squares cannot indicate the size of the residual error, so the normalized value $\tilde{e}_{S S E}=\frac{e_{S S E}}{\bar{x}}$ is used, where $\bar{x}$ is the mean value of the group of data points.

\section{Conclusion}

Aiming at the diagnosis of spaceborne ionospheric plasma, we developed a new type of Langmuir probe instrument that is described in this paper. From the theoretical design to the experimental verification, it is demonstrated that this instrument can diagnose plasmas in the density range from $10^{8}$ to $10^{13} \mathrm{~m}^{-3}$. It not only covers the normal range of plasma density in the ionosphere but can also detect abnormal ionospheric disturbances caused by solar activity. Its novel acquisition circuit can ensure the collection of a complete $I-V$ characteristic curve to obtain physical parameters such as electron temperature, plasma potential, and plasma density. Its third-order LPF circuit, composed of a single amplifier, resistances, and capacitances, can effectively attenuate the high-frequency noise doped in the probe DC signal, which significantly improves the SNR of the collected signal to above $120 \mathrm{~dB}$. The printed circuit board of the instrument is designed by circuit CAD software, and a polynomial fitting algorithm is used to 
trace back the collected current. Experiments using the proposed instrument over an extensive plasma density range were carried out in the Space Plasma Simulation Chamber of the SPDL, and promising experimental results were obtained by analyzing and comparing the data collected by SMU and NCAD.

The launch cost of micro-nano satellites is decreasing, and the number of launches is increasing year after year. A small size, low power consumption, and diversified functions are the particular requirements of micro-nano satellite payloads. The plasma diagnosis instrument developed in this study can realize the high-precision diagnosis of space plasma and is designed for the payload of micro-satellites. It can realize a variety of space plasma detection tasks and meet the increasing demand for space weather detection.

\section{Acknowledgments}

This work was supported by the National Natural Science Foundation of China (Grant No. 42120104003 and 41874170). All the experiments were carried out in the SPDL of Shandong University. We would like to extend our deep gratitude to all the researchers, engineers, and students who have contributed to the construction of the laboratory.

\section{References}

1 P. M. Kintner and C. E. Seyler: SSRv 41 (1985) 91. https://doi.org/10.1007/BF00241347

2 N. A. Gondarenko and P. N. Guzdar: Geophys. Res. Lett. 31 (2004) 11802. https://doi.org/10.1029/2004g1019703

3 N. A. Gondarenko and P. N. Guzdar: Geophys. Res. Lett. 33 (2006) 22107. https://doi.org/https://doi. org/10.1029/2006GL028033

4 C. L. Rino: Radio Sci. 14 (1979) 1135. https://doi.org/10.1029/RS014i006p01135

5 R. L. Boggess, L. H. Brace, and N. W. Spencer: J. Geophys. Res. 64 (1959) 1627. https://doi.org/10.1029/ JZ064i010p01627

6 B. H. Subbaraya, S. Prakash, S. P. Gupta, and H. S. S. Sinha: Planet. Space Sci. 22 (1974) 180. https://doi. org/10.1016/0032-0633(74)90133-0

7 R. F. Woodman and C. La Hoz: J. Geophys. Res. 81 (1976) 5447. https://doi.org/10.1029/JA081i031p05447

8 L. H. Brace: In Measurement Techniques in Space Plasmas: Particles (American Geophysical Union, New York, 1998) 2nd ed., Chap. 3.

9 H. M. Mott-Smith and I. Langmuir: Phys. Rev. 28 (1926) 727. https://doi.org/10.1103/PhysRev.28.727

10 H. Hoang, K. Røed, T. A. Bekkeng, J. I. Moen, A. Spicher, L. B. N. Clausen, W. J. Miloch, E. Trondsen, and A. Pedersen: MeScT 29 (2018) 065906. https://doi.org/10.1088/1361-6501/aab948

11 H. Hoang, L. B. N. Clausen, K. Røed, T. A. Bekkeng, E. Trondsen, B. Lybekk, H. Strøm, D. M. Bang-Hauge, A. Pedersen, A. Spicher, and J. I. Moen: SSRv 214 (2018) 75. https://doi.org/10.1007/s11214-018-0509-2

12 H. Hoang, K. Røed, T. A. Bekkeng, J. I. Moen, L. B. N. Clausen, E. Trondsen, B. Lybekk, H. Strøm, D. M. Bang-Hauge, A. Pedersen, C. D. A. Nokes, C. Cupido, I. R. Mann, M. Ariel, D. Portnoy, and E. Sagi: SSRv 215 (2019) 21. https://doi.org/10.1007/s11214-019-0586-X

13 C. Li, Y. B. Guan, A. B. Zhang, X. Z. Zhang, and Y. Q. Sun: AcPSn 65 (2016) 335. https://doi.org/10.7498/ aps.65.189401.(in Chinese).

14 J. P. Lebreton, S. Stverak, P. Travnicek, M. Maksimovic, D. Klinge, S. Merikallio, D. Lagoutte, B. Poirier, P. L. Blelly, Z. Kozacek, and M. Salaquarda: Planet. Space Sci. 54 (2006) 472. https://doi.org/10.1016/j. pss.2005.10.017

15 K. I. Oyama, Y. W. Hsu, G. S. Jiang, W. H. Chen, C. Z. Cheng, H. K. Fang, and W. T. Liu: Rev. Sci. Instrum. 86 (2015) 084703. https://doi.org/10.1063/1.4927342

16 T. A. Bekkeng, E. S. Helgeby, A. Pedersen, E. Trondsen, T. Lindem, and J. I. Moen: IEEE Trans. Aerospace Electron. Syst. 55 (2019) 2951. https://doi.org/10.1109/taes.2019.2900132

17 T. A. Bekkeng, A. Barjatya, U. P. Hoppe, A. Pedersen, J. I. Moen, M. Friedrich, and M. Rapp: AnGeo 31 (2013) 187. https://doi.org/10.5194/angeo-31-187-2013 
18 W. E. Amatucci, P. W. Schuck, D. N. Walker, P. M. Kintner, S. Powell, B. Holback, and D. Leonhardt: Rev. Sci. Instrum. 72 (2001) 2052. https://doi.org/10.1063/1.1357234

19 H. Hoang, K. Røed, T. A. Bekkeng, E. Trondsen, L. B. N. Clausen, W. J. Miloch, and J. I. Moen: MeScT 28 (2017) 115903. https://doi.org/10.1088/1361-6501/aa87e1

20 K. S. Jacobsen, A. Pedersen, J. I. Moen, and T. A. Bekkeng: MeScT 21 (2010) 085902. https://doi. org/10.1088/0957-0233/21/8/085902

21 T. A. Bekkeng, K. S. Jacobsen, J. K. Bekkeng, A. Pedersen, T. Lindem, J. P. Lebreton, and J. I. Moen: MeScT 21 (2010) 085903. https://doi.org/10.1088/0957-0233/21/8/085903

22 C. Vincent, W. McCarthy, T. Golfinopoulos, B. LaBombard, R. Sharples, J. Lovell, G. Naylor, S. Hall, J. Harrison, and A. Q. Kuang: Rev. Sci. Instrum. 90 (2019) 083504. https://doi.org/10.1063/1.5109834

23 F. F. Chen: Introduction to Plasma Physics and Controlled Fusion (Springer International Publishing, Paris, 2016) 3rd ed., Chap. 2.

24 J. Wang, Q. H. Zhang, and Q. F. Du: 2020 5th Int. Conf. Mechanical, Control and Computer Engineering (IEEE, 2020) 208-211.

25 H. C. Yeh, S. Y. Su, Y. C. Yeh, J. M. Wu, R. A. Heelis, and B. J. Holt: Terr. Atmos. Ocean. Sci. 20 (1999) 19. https://doi.org/10.3319/tao.1999.10.S.19(rocsat)

26 Z.-W. Lin, C.-K. Chao, J.-Y. Liu, C.-M. Huang, Y.-H. Chu, C.-L. Su, Y.-C. Mao, and Y.-S. Chang: Terr. Atmos. Ocean. Sci. 28 (2017) 99. https://doi.org/10.3319/tao.2016.09.14.01(eof5)

27 Y. Duann, L. C. Chang, C.-K. Chao, Y.-C. Chiu, R. Tsai-Lin, T.-Y. Tai, W.-H. Luo, C.-T. Liao, H.-T. Liu, C.-J. Chung, R. Duann, C.-L. Kuo, J.-Y. Liu, Z.-M. Yang, G. F. Gacal, A. Chandran, H. Priyardarshan, A. Verma, T.-W. Fang, and S. Srivastava: AdSpR 66 (2020) 116. https://doi.org/10.1016/j.asr.2020.01.012

28 X. Wang, C. T. Howes, M. Horanyi, and S. Robertson: Rev. Sci. Instrum. 84 (2013) 013506. https://doi. org/10.1063/1.4776201 